2015 Управление, вычислительная техника и информатика № 1 (30)

\title{
Л.А. Нежельская
}

\section{СОВМЕСТНАЯ ПЛОТНОСТЬ ВЕРОЯТНОСТЕЙ ДЛИТЕЛЬНОСТИ ИНТЕРВАЛОВ МОДУЛИРОВАННОГО МАР-ПОТОКА СОБЫТИЙ И УСЛОВИЯ РЕКУРРЕНТНОСТИ ПОТОКА}

\begin{abstract}
Изучается модулированный МАР-поток событий, являющийся одной из адекватных математических моделей информационных потоков событий в цифровых сетях интегрального обслуживания (ISDN). Приводятся явный вид плотности вероятностей длительности интервала между моментами наступления соседних событий потока, а также явный вид совместной плотности вероятностей длительности двух соседних интервалов. Рассматриваются условия рекуррентности потока.

Ключевые слова: модулированный МАР-поток событий; инфинитезимальные характеристики; плотность вероятностей; совместная плотность вероятностей; рекуррентность потока.
\end{abstract}

Интенсивное развитие компьютерной техники и информационных технологий во многом определило важную сферу приложений теории массового обслуживания - проектирование и создание информационно-вычислительных сетей, телекоммуникационных сетей, которые называют цифровыми сетями интегрального обслуживания - Integrated Service Digital Networks (ISDN). Всё это послужило стимулом к созданию адекватных математических моделей реальных информационных потоков, функционирующих в ISDN, так называемых дважды стохастических потоков событий. В работе [1] дважды стохастический поток определяется как случайный поток событий с интенсивностью, представляющей собой случайный процесс. Дважды стохастические потоки делятся на два класса: к первому классу относятся потоки, интенсивность которых есть непрерывный случайный процесс; ко второму классу относятся потоки, интенсивность которых есть кусочно-постоянный случайный процесс с конечным числом состояний. Второй класс потоков впервые введен в рассмотрение практически одновременно в 1979 г. в статьях [2-4]. В [2, 3] данные потоки названы MC (Markov chain)-потоками; в [4] - MVP (Markov versatile processes)-потоками. Последние начиная с конца 1980-х гг. носят название MAP (Markovian arrival process)-потоков событий. МАР-потоки событий наиболее характерны при описании информационных потоков в реальных телекоммуникационных сетях [5]. В зависимости от смены состояний МС-потоки можно разделить на три типа: 1) синхронные потоки событий [6-11]; 2) асинхронные и обобщённые асинхронные потоки событий [12-17]; 3) полусинхронные и обобщённые полусинхронные потоки событий [18-23]. В [24] введены в рассмотрение МАР-потоки событий первого порядка (собственно МАРпотоки, введенные в [4]) и МАР-потоки событий второго порядка (синхронизированная суперпозиция двух МАР-потоков первого порядка, отличающихся друг от друга исходными параметрами). В [24] показано, что синхронный МС-поток является частным случаем МАР-потока первого порядка; асинхронный, обобщённый асинхронный, полусинхронный и обобщённый полусинхронный МС-потоки являются частным случаем МАР-потока второго порядка.

Режим функционирования системы массового обслуживания непосредственно зависит от параметров МС (МАР)-потока и состояний, в которых находится поток. В реальных ситуациях параметры входящих потоков событий, как правило, неизвестны, либо частично известны, либо (что ещё более ухудшает ситуацию) изменяются со временем. Вследствие этого важными задачами являются задачи оценки в произвольный момент времени состояний $[14,17,19,23,25]$ и параметров [6-12, 16, 18, 20-22] потока по наблюдениям за этим потоком.

Для решения задачи оценивания (тем или иным статистическим методом) параметров потока необходимо знать вероятностные свойства потока $[13,15,26]$. В настоящей статье рассматривается модулированный МАР-поток событий (относится к классу МАР-потоков событий второго порядка), введённый в работах [27-29] и являющийся обобщением МАР-потока первого порядка [24, 25]. Находятся явные виды плотности вероятностей значений длительности интервала между моментами наступления 
соседних событий потока и совместной плотности вероятностей значений длительности двух соседних интервалов.

\section{1. Постановка задачи}

Рассматривается модулированный МАР-поток событий с интенсивностью, представляющей собой кусочно-постоянный стационарный случайный процесс $\lambda(t)$ с двумя состояниями: $\lambda(t)=\lambda_{1}$ либо $\lambda(t)=\lambda_{2}\left(\lambda_{1}>\lambda_{2} \geq 0\right)$. Длительность пребывания процесса $\lambda(t)$ в $i$-м состоянии зависит от двух случайных величин: 1) первая случайная величина распределена по экспоненциальному закону $F_{i}^{(1)}(t)=1-e^{-\alpha_{i} t}$, $i=1,2$; в момент окончания $i$-го состояния процесс $\lambda(t)$ переходит с вероятностью единица из $i$-го состояния в $j$-е, $i, j=1,2(i \neq j) ; 2)$ вторая случайная величина распределена по экспоненциальному закону $F_{i}^{(2)}(t)=1-e^{-\lambda_{i} t}, i=1,2$; в момент окончания $i$-го состояния процесс $\lambda(t)$ переходит с вероятностью $P_{1}\left(\lambda_{j} \mid \lambda_{i}\right)$ в $j$-е состояние $(i \neq j)$ с наступлением события, либо с вероятностью $P_{0}\left(\lambda_{j} \mid \lambda_{i}\right)$ переходит в $j$-е состояние $(i \neq j)$ без наступления события, либо с вероятностью $P_{1}\left(\lambda_{i} \mid \lambda_{i}\right)$ переходит в $i$-е состояние с наступлением события. При этом $P_{0}\left(\lambda_{j} \mid \lambda_{i}\right)+P_{1}\left(\lambda_{j} \mid \lambda_{i}\right)+P_{1}\left(\lambda_{i} \mid \lambda_{i}\right)=1$. Случайные величины являются независимыми друг от друга. В сделанных предположениях $\lambda(t)$ - марковский процесс. Матрицы инфинитезимальных характеристик процесса $\lambda(t)$ примут вид

$$
D_{0}=\left\|\begin{array}{cc}
-\left(\alpha_{1}+\lambda_{1}\right) & \alpha_{1}+\lambda_{1} P_{0}\left(\lambda_{2} \mid \lambda_{1}\right) \\
\alpha_{2}+\lambda_{2} P_{0}\left(\lambda_{1} \mid \lambda_{2}\right) & -\left(\alpha_{2}+\lambda_{2}\right)
\end{array}\right\|, D_{1}=\left\|\begin{array}{cc}
\lambda_{1} P_{1}\left(\lambda_{1} \mid \lambda_{1}\right) & \lambda_{1} P_{1}\left(\lambda_{2} \mid \lambda_{1}\right) \\
\lambda_{2} P_{1}\left(\lambda_{1} \mid \lambda_{2}\right) & \lambda_{2} P_{1}\left(\lambda_{2} \mid \lambda_{2}\right)
\end{array}\right\| .
$$

Элементами матрицы $D_{1}$ являются интенсивности переходов процесса $\lambda(t)$ из состояния в состояние с наступлением события. Недиагональные элементы матрицы $D_{0}-$ интенсивности переходов из состояния в состояние без наступления события. Диагональные элементы матрицы $D_{0}-$ интенсивности выхода процесса $\lambda(t)$ из своих состояний, взятые с противоположным знаком [3]. Заметим, что в приведённом определении модулированного МАР-потока событий в явном виде не оговаривается, в каком состоянии процесса $\lambda(t)$ наступает событие потока при переходе $\lambda(t)$ из первого (второго) состояния во второе (в первое). Отметим, что в реальных потоках событий, моделями которых являются модулированные МАР-потоки, событие потока (в момент окончания того или иного состояния процесса $\lambda(t))$ наступает с полной определённостью в первом или во втором состоянии процесса $\lambda(t)$. В данной статье при получении формул для плотностей вероятностей данное обстоятельство является несущественным, так как наступление события и переход процесса $\lambda(t)$ из состояния $i$ в состояние $j, i, j=1,2$, происходят мгновенно. Вариант возникающей ситуации представлен на рис. 1 , где $\lambda_{1}$ и $\lambda_{2}-$ состояния случайного процесса $\lambda(t) ; t_{1}, t_{2}, \ldots-$ моменты наступления событий потока.

Процесс $\lambda(t)$ принципиально ненаблюдаемый, а наблюдаемыми являются только временные моменты наступления событий потока $t_{1}, t_{2}, \ldots$. Рассматривается стационарный режим функционирования потока. В силу предпосылок в моменты времени наступления событий $t_{1}, t_{2}, \ldots, t_{k}, \ldots$ последовательность $\left\{\lambda\left(t_{k}\right)\right\}$ представляет собой вложенную цепь Маркова, т.е. модулированный МАР-поток обладает марковским свойством, если его эволюцию рассматривать с момента $t_{k}$ - момента наступления события потока, $k=1,2, \ldots$.

Обозначим $\tau_{k}=t_{\mathrm{k}+1}-t_{k}, k=1,2, \ldots,-$ значение длительности $k$-го интервала между соседними событиями потока. Так как рассматривается стационарный режим, то плотность вероятностей значений длительности $k$-го интервала $p\left(\tau_{k}\right)=p(\tau), \tau \geq 0$, для любого $k$. В силу этого момент времени $t_{k}$ наступления события без ограничения общности можно положить равным нулю или, что то же самое, момент наступления события есть $\tau=0$.

Пусть $\left(t_{k}, t_{k+1}\right),\left(t_{k+1}, t_{k+2}\right)$ - два смежных интервала, длительности которых есть $\tau_{k}=t_{k+1}-t_{k}$ и $\tau_{k+1}=$ $=t_{k+2}-t_{k+1}$ соответственно; их расположение на временной оси в силу стационарности потока произвольно. Тогда, полагая $k=1$, будем рассматривать два соседних интервала $\left(t_{1}, t_{2}\right),\left(t_{2}, t_{3}\right)$ с соответствующими значениями длительностей $\tau_{1}=t_{2}-t_{1}$ и $\tau_{2}=t_{3}-t_{2} ; \tau_{1} \geq 0, \tau_{2} \geq 0$. При этом $\tau_{1}=0$ соответствует моменту $t_{1}$ наступления события потока; $\tau_{2}=0$ соответствует моменту $t_{2}$ наступления следующего события потока. Соответствующая совместная плотность вероятностей при этом есть $p\left(\tau_{1}, \tau_{2}\right), \tau_{1} \geq 0, \tau_{2} \geq 0$. 


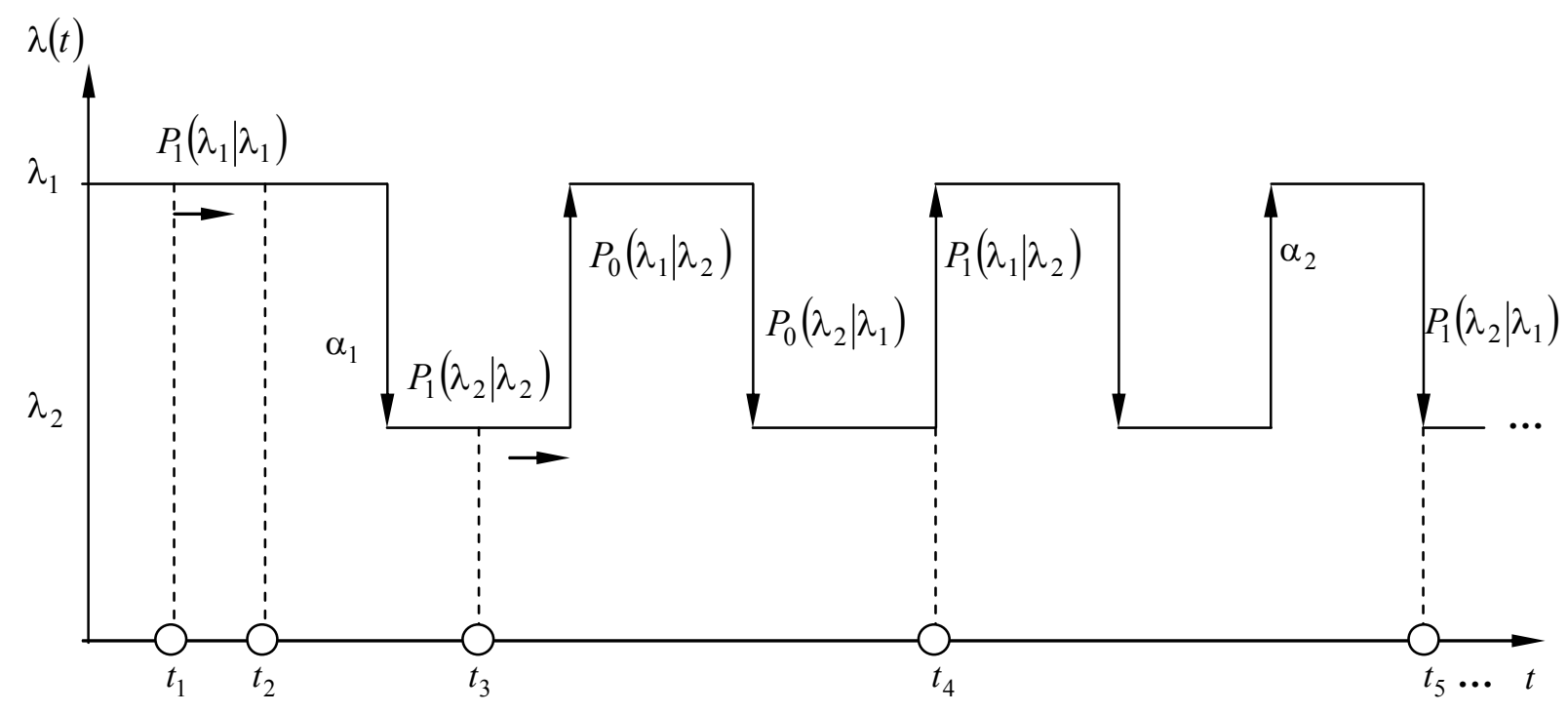

Рис. 1. Модулированный МАР-поток событий

Задача заключается в нахождении явного вида $p(\tau)$ и явного вида $p\left(\tau_{1}, \tau_{2}\right)$, а также в установлении условий рекуррентности модулированного МАР-потока событий.

\section{2. Вывод плотности вероятностей $p(\tau)$}

Введём в рассмотрение вероятности $p_{i j}(\tau)$ того, что на интервале $(0, \tau)$ нет событий модулированного МАР-потока и в момент времени $\tau$ значение процесса $\lambda(\tau)=\lambda_{j}$ при условии, что в момент времени $\tau=0$ значение процесса $\lambda(0)=\lambda_{i}, i, j=1,2$. Тогда для указанных вероятностей справедливы следующие системы дифференциальных уравнений с начальными условиями:

$$
\begin{aligned}
& \left\{\begin{array}{l}
p_{11}^{\prime}(\tau)=-\left(\alpha_{1}+\lambda_{1}\right) p_{11}(\tau)+\left(\alpha_{2}+\lambda_{2} P_{0}\left(\lambda_{1} \mid \lambda_{2}\right)\right) p_{12}(\tau), \\
p_{12}^{\prime}(\tau)=\left(\alpha_{1}+\lambda_{1} P_{0}\left(\lambda_{2} \mid \lambda_{1}\right)\right) p_{11}(\tau)-\left(\alpha_{2}+\lambda_{2}\right) p_{12}(\tau), \\
p_{11}(0)=1, p_{12}(0)=0,
\end{array}\right. \\
& \left\{\begin{array}{l}
p_{22}^{\prime}(\tau)=-\left(\alpha_{2}+\lambda_{2}\right) p_{22}(\tau)+\left(\alpha_{1}+\lambda_{1} P_{0}\left(\lambda_{2} \mid \lambda_{1}\right)\right) p_{21}(\tau), \\
p_{21}^{\prime}(\tau)=\left(\alpha_{2}+\lambda_{2} P_{0}\left(\lambda_{1} \mid \lambda_{2}\right)\right) p_{22}(\tau)-\left(\alpha_{1}+\lambda_{1}\right) p_{21}(\tau), \\
p_{22}(0)=1, p_{21}(0)=0 .
\end{array}\right.
\end{aligned}
$$

Решая записанные системы, находим вероятности $p_{i j}(\tau), i, j=1,2$, в виде

$$
\begin{gathered}
p_{11}(\tau)=\frac{1}{z_{2}-z_{1}}\left[\left(\lambda_{2}+\alpha_{2}-z_{1}\right) \mathrm{e}^{-z_{1} \tau}-\left(\lambda_{2}+\alpha_{2}-z_{2}\right) \mathrm{e}^{-z_{2} \tau}\right], \\
p_{12}(\tau)=\frac{\alpha_{1}+\lambda_{1} P_{0}\left(\lambda_{2} \mid \lambda_{1}\right)}{z_{2}-z_{1}}\left[\mathrm{e}^{-z_{1} \tau}-\mathrm{e}^{-z_{2} \tau}\right], \\
p_{22}(\tau)=\frac{1}{z_{2}-z_{1}}\left[\left(\lambda_{1}+\alpha_{1}-z_{1}\right) \mathrm{e}^{-z_{1} \tau}-\left(\lambda_{1}+\alpha_{1}-z_{2}\right) \mathrm{e}^{-z_{2} \tau}\right], \\
p_{21}(\tau)=\frac{\alpha_{2}+\lambda_{2} P_{0}\left(\lambda_{1} \mid \lambda_{2}\right)}{z_{2}-z_{1}}\left[\mathrm{e}^{-z_{1} \tau}-\mathrm{e}^{-z_{2} \tau}\right], \\
z_{1}=\frac{1}{2}\left[\left(\lambda_{1}+\lambda_{2}+\alpha_{1}+\alpha_{2}\right)-\sqrt{\left(\lambda_{1}-\lambda_{2}+\alpha_{1}-\alpha_{2}\right)^{2}+4\left(\alpha_{1}+\lambda_{1} P_{0}\left(\lambda_{2} \mid \lambda_{1}\right)\right)\left(\alpha_{2}+\lambda_{2} P_{0}\left(\lambda_{1} \mid \lambda_{2}\right)\right)}\right],
\end{gathered}
$$




$$
\begin{gathered}
z_{2}=\frac{1}{2}\left[\left(\lambda_{1}+\lambda_{2}+\alpha_{1}+\alpha_{2}\right)+\sqrt{\left(\lambda_{1}-\lambda_{2}+\alpha_{1}-\alpha_{2}\right)^{2}+4\left(\alpha_{1}+\lambda_{1} P_{0}\left(\lambda_{2} \mid \lambda_{1}\right)\right)\left(\alpha_{2}+\lambda_{2} P_{0}\left(\lambda_{1} \mid \lambda_{2}\right)\right)}\right], \\
0<z_{1}<z_{2} .
\end{gathered}
$$

С учётом определения модулированного МАР-потока введём $p_{11}(\tau) \lambda_{1} \Delta \tau P_{1}\left(\lambda_{1} \mid \lambda_{1}\right)+o(\Delta \tau)-$ совместную вероятность того, что без наступления событий потока на интервале $(0, \tau)$ процесс $\lambda(\tau)$ перешёл на этом интервале из первого состояния в первое, на полуинтервале $[\tau, \tau+\Delta \tau)$ произошло окончание первого состояния процесса $\lambda(\tau)$ и процесс $\lambda(\tau)$ на полуинтервале $[\tau, \tau+\Delta \tau)$ перешёл из первого состояния в первое с наступлением события потока. Аналогичные совместные вероятности примут вид

$$
\begin{array}{cc}
p_{12}(\tau) \lambda_{2} \Delta \tau P_{1}\left(\lambda_{1} \mid \lambda_{2}\right)+o(\Delta \tau), & p_{12}(\tau) \lambda_{2} \Delta \tau P_{1}\left(\lambda_{2} \mid \lambda_{2}\right)+o(\Delta \tau), \\
p_{21}(\tau) \lambda_{1} \Delta \tau P_{1}\left(\lambda_{1} \mid \lambda_{1}\right)+o(\Delta \tau), & p_{21}(\tau) \lambda_{1} \Delta \tau P_{1}\left(\lambda_{2} \mid \lambda_{1}\right)+o(\Delta \tau), \\
p_{22}(\tau) \lambda_{2} \Delta \tau P_{1}\left(\lambda_{1} \mid \lambda_{2}\right)+o(\Delta \tau), & p_{22}(\tau) \lambda_{2} \Delta \tau P_{1}\left(\lambda_{2} \mid \lambda_{2}\right)+o(\Delta \tau), \\
p_{11}(\tau) \lambda_{1} \Delta \tau P_{1}\left(\lambda_{2} \mid \lambda_{1}\right)+o(\Delta \tau) .
\end{array}
$$

Тогда соответствующие плотности вероятностей запишутся в виде

$$
\begin{array}{ll}
\tilde{p}_{11}{ }^{(1)}(\tau)=\lambda_{1} P_{1}\left(\lambda_{1} \mid \lambda_{1}\right) p_{11}(\tau), & \tilde{p}_{11}{ }^{(2)}(\tau)=\lambda_{2} P_{1}\left(\lambda_{1} \mid \lambda_{2}\right) p_{12}(\tau), \\
\tilde{p}_{12}{ }^{(1)}(\tau)=\lambda_{2} P_{1}\left(\lambda_{2} \mid \lambda_{2}\right) p_{12}(\tau), & \tilde{p}_{12}{ }^{(2)}(\tau)=\lambda_{1} P_{1}\left(\lambda_{2} \mid \lambda_{1}\right) p_{11}(\tau), \\
\tilde{p}_{21}{ }^{(1)}(\tau)=\lambda_{1} P_{1}\left(\lambda_{1} \mid \lambda_{1}\right) p_{21}(\tau), & \tilde{p}_{21}{ }^{(2)}(\tau)=\lambda_{2} P_{1}\left(\lambda_{1} \mid \lambda_{2}\right) p_{22}(\tau), \\
\tilde{p}_{22}{ }^{(1)}(\tau)=\lambda_{2} P_{1}\left(\lambda_{2} \mid \lambda_{2}\right) p_{22}(\tau), & \tilde{p}_{22}{ }^{(2)}(\tau)=\lambda_{1} P_{1}\left(\lambda_{2} \mid \lambda_{1}\right) p_{21}(\tau) .
\end{array}
$$

Очевидно, что плотности вероятностей $\tilde{p}_{i j}(\tau)$ того, что без наступления событий потока на интервале $(0, \tau)$ и наступления события в момент $\tau$ процесс $\lambda(\tau)$ перейдёт на этом интервале из состояния $i$ в состояние $j(i, j=1,2)$, запишутся в виде

$$
\begin{aligned}
& \tilde{p}_{11}(\tau)=\lambda_{1} P_{1}\left(\lambda_{1} \mid \lambda_{1}\right) p_{11}(\tau)+\lambda_{2} P_{1}\left(\lambda_{1} \mid \lambda_{2}\right) p_{12}(\tau), \\
& \tilde{p}_{12}(\tau)=\lambda_{2} P_{1}\left(\lambda_{2} \mid \lambda_{2}\right) p_{12}(\tau)+\lambda_{1} P_{1}\left(\lambda_{2} \mid \lambda_{1}\right) p_{11}(\tau), \\
& \tilde{p}_{21}(\tau)=\lambda_{1} P_{1}\left(\lambda_{1} \mid \lambda_{1}\right) p_{21}(\tau)+\lambda_{2} P_{1}\left(\lambda_{1} \mid \lambda_{2}\right) p_{22}(\tau), \\
& \tilde{p}_{22}(\tau)=\lambda_{2} P_{1}\left(\lambda_{2} \mid \lambda_{2}\right) p_{22}(\tau)+\lambda_{1} P_{1}\left(\lambda_{2} \mid \lambda_{1}\right) p_{21}(\tau) .
\end{aligned}
$$

Подставляя (1) в (2), получаем выражения для плотностей вероятностей $\tilde{p}_{i j}(\tau), i, j=1,2$, в явном виде.

Поскольку $\tau$ - произвольный момент времени, то $p_{i j}$ - вероятности перехода процесса $\lambda(\tau)$ из состояния $i$ в состояние $j(i, j=1,2)$ за время, которое пройдёт от момента $\tau=0$ до момента наступления очередного события потока, определятся в виде

$$
\begin{aligned}
& p_{11}=\int_{0}^{\infty} \tilde{p}_{11}(\tau) d \tau=\lambda_{1} P_{1}\left(\lambda_{1} \mid \lambda_{1}\right) \int_{0}^{\infty} p_{11}(\tau) d \tau+\lambda_{2} P_{1}\left(\lambda_{1} \mid \lambda_{2}\right) \int_{0}^{\infty} p_{12}(\tau) d \tau, \\
& p_{12}=\int_{0}^{\infty} \tilde{p}_{12}(\tau) d \tau=\lambda_{2} P_{1}\left(\lambda_{2} \mid \lambda_{2}\right) \int_{0}^{\infty} p_{12}(\tau) d \tau+\lambda_{1} P_{1}\left(\lambda_{2} \mid \lambda_{1}\right) \int_{0}^{\infty} p_{11}(\tau) d \tau, \\
& p_{21}=\int_{0}^{\infty} \tilde{p}_{21}(\tau) d \tau=\lambda_{1} P_{1}\left(\lambda_{1} \mid \lambda_{1}\right) \int_{0}^{\infty} p_{21}(\tau) d \tau+\lambda_{2} P_{1}\left(\lambda_{1} \mid \lambda_{2}\right) \int_{0}^{\infty} p_{22}(\tau) d \tau, \\
& p_{22}=\int_{0}^{\infty} \tilde{p}_{22}(\tau) d \tau=\lambda_{2} P_{1}\left(\lambda_{2} \mid \lambda_{2}\right) \int_{0}^{\infty} p_{22}(\tau) d \tau+\lambda_{1} P_{1}\left(\lambda_{2} \mid \lambda_{1}\right) \int_{0}^{\infty} p_{21}(\tau) d \tau .
\end{aligned}
$$

Подставляя (1) в (3), находим

$$
\begin{aligned}
& p_{11}=\left(z_{1} z_{2}\right)^{-1}\left[\lambda_{1} P_{1}\left(\lambda_{1} \mid \lambda_{1}\right)\left(\lambda_{2}+\alpha_{2}\right)+\lambda_{2} P_{1}\left(\lambda_{1} \mid \lambda_{2}\right)\left(\alpha_{1}+\lambda_{1} P_{0}\left(\lambda_{2} \mid \lambda_{1}\right)\right)\right], \\
& p_{12}=\left(z_{1} z_{2}\right)^{-1}\left[\lambda_{1} P_{1}\left(\lambda_{2} \mid \lambda_{1}\right)\left(\lambda_{2}+\alpha_{2}\right)+\lambda_{2} P_{1}\left(\lambda_{2} \mid \lambda_{2}\right)\left(\alpha_{1}+\lambda_{1} P_{0}\left(\lambda_{2} \mid \lambda_{1}\right)\right)\right], \\
& p_{21}=\left(z_{1} z_{2}\right)^{-1}\left[\lambda_{2} P_{1}\left(\lambda_{1} \mid \lambda_{2}\right)\left(\lambda_{1}+\alpha_{1}\right)+\lambda_{1} P_{1}\left(\lambda_{1} \mid \lambda_{1}\right)\left(\alpha_{2}+\lambda_{2} P_{0}\left(\lambda_{1} \mid \lambda_{2}\right)\right)\right],
\end{aligned}
$$




$$
\begin{gathered}
p_{22}=\left(z_{1} z_{2}\right)^{-1}\left[\lambda_{2} P_{1}\left(\lambda_{2} \mid \lambda_{2}\right)\left(\lambda_{1}+\alpha_{1}\right)+\lambda_{1} P_{1}\left(\lambda_{2} \mid \lambda_{1}\right)\left(\alpha_{2}+\lambda_{2} P_{0}\left(\lambda_{1} \mid \lambda_{2}\right)\right)\right], \\
z_{1} z_{2}=\left(\lambda_{1}+\alpha_{1}\right)\left(\lambda_{2}+\alpha_{2}\right)-\left(\alpha_{1}+\lambda_{1} P_{0}\left(\lambda_{2} \mid \lambda_{1}\right)\right)\left(\alpha_{2}+\lambda_{2} P_{0}\left(\lambda_{1} \mid \lambda_{2}\right)\right) .
\end{gathered}
$$

Введём в рассмотрение $\pi_{i}(0)$ - условную стационарную вероятность того, что процесс $\lambda(\tau)$ в момент времени $\tau=0$ находится в состоянии $i$ при условии, что в момент времени $\tau=0$ событие потока наступило, $i=1,2\left(\pi_{1}(0)+\pi_{2}(0)=1\right)$. Тогда, так как $\left\{\lambda\left(t_{k}\right)\right\}$ есть вложенная цепь Маркова, то для вероятностей $\pi_{i}(0)$ справедливы следующие уравнения:

$$
\pi_{1}(0)=p_{11} \pi_{1}(0)+p_{21} \pi_{2}(0), \quad \pi_{2}(0)=p_{12} \pi_{1}(0)+p_{22} \pi_{2}(0),
$$

где переходные вероятности $p_{i j}(i, j=1,2)$ определены формулами (4).

Подставляя (4) в (5), получаем

$$
\begin{aligned}
& \pi_{1}(0)=\left\{\lambda_{2} P_{1}\left(\lambda_{1} \mid \lambda_{2}\right)\left(\lambda_{1}+\alpha_{1}\right)+\lambda_{1} P_{1}\left(\lambda_{1} \mid \lambda_{1}\right)\left(\alpha_{2}+\lambda_{2} P_{0}\left(\lambda_{1} \mid \lambda_{2}\right)\right)\right\}\left\{\lambda_{1} P_{1}\left(\lambda_{2} \mid \lambda_{1}\right)\left(\lambda_{2}+\alpha_{2}\right)+\right. \\
& \left.+\lambda_{2} P_{1}\left(\lambda_{1} \mid \lambda_{2}\right)\left(\lambda_{1}+\alpha_{1}\right)+\lambda_{1} P_{1}\left(\lambda_{1} \mid \lambda_{1}\right)\left(\alpha_{2}+\lambda_{2} P_{0}\left(\lambda_{1} \mid \lambda_{2}\right)\right)+\lambda_{2} P_{1}\left(\lambda_{2} \mid \lambda_{2}\right)\left(\alpha_{1}+\lambda_{1} P_{0}\left(\lambda_{2} \mid \lambda_{1}\right)\right)\right\}^{-1}, \\
& \pi_{2}(0)=\left\{\lambda_{1} P_{1}\left(\lambda_{2} \mid \lambda_{1}\right)\left(\lambda_{2}+\alpha_{2}\right)+\lambda_{2} P_{1}\left(\lambda_{2} \mid \lambda_{2}\right)\left(\alpha_{1}+\lambda_{1} P_{0}\left(\lambda_{2} \mid \lambda_{1}\right)\right)\right\}\left\{\lambda_{1} P_{1}\left(\lambda_{2} \mid \lambda_{1}\right)\left(\lambda_{2}+\alpha_{2}\right)+\right. \\
& \left.+\lambda_{2} P_{1}\left(\lambda_{1} \mid \lambda_{2}\right)\left(\lambda_{1}+\alpha_{1}\right)+\lambda_{1} P_{1}\left(\lambda_{1} \mid \lambda_{1}\right)\left(\alpha_{2}+\lambda_{2} P_{0}\left(\lambda_{1} \mid \lambda_{2}\right)\right)+\lambda_{2} P_{1}\left(\lambda_{2} \mid \lambda_{2}\right)\left(\alpha_{1}+\lambda_{1} P_{0}\left(\lambda_{2} \mid \lambda_{1}\right)\right)\right\}^{-1} .
\end{aligned}
$$

Плотность вероятностей $p(\tau)$ длительности интервала между соседними событиями в модулированном МАР-потоке примет вид

$$
p(\tau)=\sum_{i=1}^{2} \pi_{i}(0) \sum_{j=1}^{2} \widetilde{p}_{i j}(\tau), \quad \tau \geq 0 .
$$

Подставляя в (7) сначала (2), затем (1) и (6) и проделывая достаточно трудоёмкие преобразования, получаем явный вид плотности вероятностей $p(\tau)$ :

$$
\begin{gathered}
p(\tau)=\gamma z_{1} e^{-z_{1} \tau}+(1-\gamma) z_{2} e^{-z_{2} \tau}, \quad \tau \geq 0, \\
\gamma=\frac{1}{z_{2}-z_{1}}\left\{z_{2}-\lambda_{1} \pi_{1}(0)\left[1-P_{0}\left(\lambda_{2} \mid \lambda_{1}\right)\right]-\lambda_{2} \pi_{2}(0)\left[1-P_{0}\left(\lambda_{1} \mid \lambda_{2}\right)\right]\right\},
\end{gathered}
$$

где $z_{1}$ и $z_{2}$ определены в $(1), \pi_{1}(0)$ и $\pi_{2}(0)$ определены в (6). Положив в $z_{1}$ и $z_{2}$ параметры $\alpha_{1}=\alpha_{2}=0$, получаем плотность вероятностей $p(\tau)$ для МАР-потока [26].

\section{3. Вывод совместной плотности вероятностей $p\left(\tau_{1}, \tau_{2}\right)$}

В моменты времени наступления событий $t_{1}, t_{2}, \ldots, t_{k}, \ldots$ последовательность $\left\{\lambda\left(t_{k}\right)\right\}$ представляет собой вложенную цепь Маркова, поэтому совместная плотность вероятностей значений длительности двух соседних интервалов $p\left(\tau_{1}, \tau_{2}\right)$ примет вид

$$
p\left(\tau_{1}, \tau_{2}\right)=\sum_{i=1}^{2} \pi_{i}(0) \sum_{j=1}^{2} \widetilde{p}_{i j}\left(\tau_{1}\right) \sum_{k=1}^{2} \widetilde{p}_{j k}\left(\tau_{2}\right),
$$

где $\widetilde{p}_{i j}\left(\tau_{1}\right), \widetilde{p}_{j k}\left(\tau_{2}\right)$ - плотности вероятностей, соответствующие переходным вероятностям $p_{i j}\left(\tau_{1}\right)$, $p_{j k}\left(\tau_{2}\right)$ и вычисленные по формулам (2) при $\tau=\tau_{1}$ и $\tau=\tau_{2}$. Подставляя в (9) сначала $\widetilde{p}_{i j}\left(\tau_{1}\right), \widetilde{p}_{j k}\left(\tau_{2}\right)$, затем $p_{i j}\left(\tau_{1}\right), p_{j k}\left(\tau_{2}\right)$, определённые формулами (1) при $\tau=\tau_{1}$ и $\tau=\tau_{2}$ и, наконец, $\pi_{i}(0), i=1,2$, определенные в (6), и проделывая необходимые достаточно трудоёмкие преобразования, находим

$$
\begin{gathered}
p\left(\tau_{1}, \tau_{2}\right)=p\left(\tau_{1}\right) p\left(\tau_{2}\right)+\gamma(1-\gamma) \frac{\lambda_{1} \lambda_{2}}{z_{1} z_{2}}\left[P_{1}\left(\lambda_{1} \mid \lambda_{1}\right) P_{1}\left(\lambda_{2} \mid \lambda_{2}\right)-P_{1}\left(\lambda_{1} \mid \lambda_{2}\right) P_{1}\left(\lambda_{2} \mid \lambda_{1}\right)\right] \times \\
\times\left(z_{1} e^{-z_{1} \tau_{1}}-z_{2} e^{-z_{2} \tau_{1}}\right)\left(z_{1} e^{-z_{1} \tau_{2}}-z_{2} e^{-z_{2} \tau_{2}}\right), \quad \tau_{1} \geq 0, \tau_{2} \geq 0,
\end{gathered}
$$

где $p\left(\tau_{1}\right), p\left(\tau_{2}\right), \gamma$ определены в (8) для $\tau=\tau_{1}$ и $\tau=\tau_{2} ; z_{1}$ и $z_{2}$ определены в (1).

Полагая в (10) параметры $\alpha_{1}=\alpha_{2}=0$, получим совместную плотность вероятностей $p\left(\tau_{1}, \tau_{2}\right)$ для МАР-потока событий [26]. 


\section{4. Условия рекуррентности модулированного МАР-потока событий}

Рассмотрим случаи, при которых модулированный МАР-поток событий становится рекуррентным. С учётом выражения (8) для $\gamma$ и выражений (6) для $\pi_{i}(0), i=1,2$, находим

$$
\begin{gathered}
\gamma(1-\gamma)=\frac{z_{1} z_{2}}{\left(z_{2}-z_{1}\right)^{2}}\left(\lambda_{1}\left[1-P_{0}\left(\lambda_{2} \mid \lambda_{1}\right)\right]-\lambda_{2}\left[1-P_{0}\left(\lambda_{1} \mid \lambda_{2}\right)\right]\right) \times \\
\times\left\{\pi_{1}(0)\left(\lambda_{1}\left[1-P_{1}\left(\lambda_{1} \mid \lambda_{1}\right)\right]+\alpha_{1}\right)-\pi_{2}(0)\left(\lambda_{2}\left[1-P_{1}\left(\lambda_{2} \mid \lambda_{2}\right)\right]+\alpha_{2}\right)\right\} \times \\
\times\left\{\lambda_{1}\left[1-P_{0}\left(\lambda_{2} \mid \lambda_{1}\right)\right]\left(\lambda_{2}\left[1-P_{1}\left(\lambda_{2} \mid \lambda_{2}\right)\right]+\alpha_{2}\right)+\lambda_{2}\left[1-P_{0}\left(\lambda_{1} \mid \lambda_{2}\right)\right]\left(\lambda_{1}\left[1-P_{1}\left(\lambda_{1} \mid \lambda_{1}\right)\right]+\alpha_{1}\right)\right\}^{-1} .
\end{gathered}
$$

Анализируя выражение для $\gamma(1-\gamma)$, замечаем:

1) если $\lambda_{1}\left[1-P_{0}\left(\lambda_{2} \mid \lambda_{1}\right)\right]-\lambda_{2}\left[1-P_{0}\left(\lambda_{1} \mid \lambda_{2}\right)\right]=0$, то совместная плотность (10) факторизуется: $p\left(\tau_{1}, \tau_{2}\right)=p\left(\tau_{1}\right) p\left(\tau_{2}\right)$; подставляя указанные условия в выражение (1) для $z_{1}$, находим $z_{1}=\lambda_{1}\left[1-P_{0}\left(\lambda_{2} \mid \lambda_{1}\right)\right]$ либо $z_{1}=\lambda_{2}\left[1-P_{0}\left(\lambda_{1} \mid \lambda_{2}\right)\right]$; при этом из (8) следует, что $\gamma=1$ и $p\left(\tau_{i}\right)=z_{1} e^{-z_{1} \tau_{i}}, \tau_{i} \geq 0, i=1,2$, или $p(\tau)=z_{1} e^{-z_{1} \tau}, \tau \geq 0$;

2) если $\pi_{1}(0)\left(\lambda_{1}\left[1-P_{1}\left(\lambda_{1} \mid \lambda_{1}\right)\right]+\alpha_{1}\right)-\pi_{2}(0)\left(\lambda_{2}\left[1-P_{1}\left(\lambda_{2} \mid \lambda_{2}\right)\right]+\alpha_{2}\right)=0$, то совместная плотность (10) факторизуется: $p\left(\tau_{1}, \tau_{2}\right)=p\left(\tau_{1}\right) p\left(\tau_{2}\right)$. При этом из (1) следует, что

$$
z_{1}=\frac{\lambda_{1} \lambda_{2}\left[P_{1}\left(\lambda_{1} \mid \lambda_{2}\right)+P_{1}\left(\lambda_{1} \mid \lambda_{1}\right) P_{0}\left(\lambda_{1} \mid \lambda_{2}\right)\right]+\lambda_{1} \alpha_{2} P_{1}\left(\lambda_{1} \mid \lambda_{1}\right)+\lambda_{2} \alpha_{1} P_{1}\left(\lambda_{1} \mid \lambda_{2}\right)}{\lambda_{2}\left[1-P_{1}\left(\lambda_{2} \mid \lambda_{2}\right)\right]+\alpha_{2}}
$$

либо

$$
z_{1}=\frac{\lambda_{1} \lambda_{2}\left[P_{1}\left(\lambda_{2} \mid \lambda_{1}\right)+P_{1}\left(\lambda_{2} \mid \lambda_{2}\right) P_{0}\left(\lambda_{2} \mid \lambda_{1}\right)\right]+\lambda_{1} \alpha_{2} P_{1}\left(\lambda_{2} \mid \lambda_{1}\right)+\lambda_{2} \alpha_{1} P_{1}\left(\lambda_{2} \mid \lambda_{2}\right)}{\lambda_{1}\left[1-P_{1}\left(\lambda_{1} \mid \lambda_{1}\right)\right]+\alpha_{1}},
$$

и из (8) находим $\gamma=1$ и $p\left(\tau_{i}\right)=z_{1} e^{-z_{1} \tau_{i}}, \tau_{i} \geq 0, i=1,2$, т.е. $p(\tau)=z_{1} e^{-z_{1} \tau}, \tau \geq 0$.

Из выражения (10) для совместной плотности вероятностей $p\left(\tau_{1}, \tau_{2}\right)$ следует третье условие её факторизации: $P_{1}\left(\lambda_{1} \mid \lambda_{1}\right) P_{1}\left(\lambda_{2} \mid \lambda_{2}\right)-P_{1}\left(\lambda_{1} \mid \lambda_{2}\right) P_{1}\left(\lambda_{2} \mid \lambda_{1}\right)=0$. Тогда из (6) в результате необходимых преобразований находим

$$
\begin{aligned}
& \pi_{1}(0)=\frac{P_{1}\left(\lambda_{1} \mid \lambda_{1}\right)}{P_{1}\left(\lambda_{1} \mid \lambda_{1}\right)+P_{1}\left(\lambda_{2} \mid \lambda_{1}\right)} \text { либо } \pi_{1}(0)=\frac{P_{1}\left(\lambda_{1} \mid \lambda_{2}\right)}{P_{1}\left(\lambda_{1} \mid \lambda_{2}\right)+P_{1}\left(\lambda_{2} \mid \lambda_{2}\right)}, \\
& \pi_{2}(0)=\frac{P_{1}\left(\lambda_{2} \mid \lambda_{1}\right)}{P_{1}\left(\lambda_{1} \mid \lambda_{1}\right)+P_{1}\left(\lambda_{2} \mid \lambda_{1}\right)} \text { либо } \pi_{1}(0)=\frac{P_{1}\left(\lambda_{2} \mid \lambda_{2}\right)}{P_{1}\left(\lambda_{1} \mid \lambda_{2}\right)+P_{1}\left(\lambda_{2} \mid \lambda_{2}\right)} .
\end{aligned}
$$

Тогда из (8) следует

$$
\begin{gathered}
\gamma=\frac{1}{z_{2}-z_{1}}\left\{z_{2}-\lambda_{1} P_{1}\left(\lambda_{1} \mid \lambda_{1}\right)-\lambda_{2} P_{1}\left(\lambda_{2} \mid \lambda_{2}\right)\right\}, \\
1-\gamma=\frac{1}{z_{2}-z_{1}}\left\{-z_{1}+\lambda_{1} P_{1}\left(\lambda_{1} \mid \lambda_{1}\right)+\lambda_{2} P_{1}\left(\lambda_{2} \mid \lambda_{2}\right)\right\}
\end{gathered}
$$

и $p\left(\tau_{i}\right)=\gamma z_{1} e^{-z_{1} \tau_{i}}+(1-\gamma) z_{2} e^{-z_{2} \tau_{i}}, \tau_{i} \geq 0, i=1,2$, т.е. $p(\tau)=\gamma z_{1} e^{-z_{1} \tau}+(1-\gamma) z_{2} e^{-z_{2} \tau}, \tau \geq 0$.

Если выполняется одно из перечисленных условий, то тогда модулированный МАР-поток событий будет рекуррентным потоком. Действительно, пусть $p\left(\tau_{1}, \tau_{2}, \ldots, \tau_{k}, \tau_{k+1}\right)-$ совместная плотность вероятностей значений длительностей интервалов $\tau_{1}, \tau_{2}, \ldots, \tau_{k+1}$, где $\tau_{k}=t_{k+1}-t_{k}, k=1,2, \ldots$. Для $k=1$ имеет место $p\left(\tau_{1}, \tau_{2}\right)=p\left(\tau_{1}\right) p\left(\tau_{2}\right)$. Докажем факторизацию плотности $p\left(\tau_{1}, \tau_{2}, \ldots, \tau_{k}\right)$ методом математической индукции. Пусть $p\left(\tau_{1}, \tau_{2}, \ldots, \tau_{k}\right)=p\left(\tau_{1}\right) \ldots p\left(\tau_{k}\right)$. Так как в моменты наступления событий потока $t_{1}, t_{2}, \ldots, t_{k}$ последовательность $\left\{\lambda\left(t_{k}\right)\right\}, k=1,2, \ldots$, образует вложенную цепь Маркова, то дальнейшее после момента $t_{k}$ поведение потока не зависит от предыстории. Тогда $p\left(\tau_{1}, \ldots, \tau_{k}, \tau_{k+1}\right)=p\left(\tau_{k+1} \mid \tau_{1}, \ldots, \tau_{k}\right) p\left(\tau_{1}, \ldots, \tau_{k}\right)=$ $=p\left(\tau_{k+1} \mid \tau_{k}\right) p\left(\tau_{1}, \ldots, \tau_{k}\right)$. Здесь $p\left(\tau_{k+1} \mid \tau_{k}\right)=p\left(\tau_{k}, \tau_{k+1}\right) \mid p\left(\tau_{k}\right)$. Так как для двух соседних интервалов $\left(t_{k}, t_{k+1}\right)$, 
$\left(t_{k+1}, t_{k+2}\right), k=1,2, \ldots$, расположенных на временной оси произвольно, справедливо $p\left(\tau_{k}, \tau_{k+1}\right)=p\left(\tau_{k}\right) p\left(\tau_{k+1}\right)$, то $p\left(\tau_{k+1} \mid \tau_{k}\right)=p\left(\tau_{k+1}\right)$. Таким образом, $p\left(\tau_{1}, \ldots, \tau_{k}, \tau_{k+1}\right)=p\left(\tau_{k+1}\right) p\left(\tau_{1}, \ldots \tau_{k}\right)$ или $p\left(\tau_{1}, \ldots, \tau_{k}, \tau_{k+1}\right)=p\left(\tau_{1}\right) p\left(\tau_{2}\right) \ldots p\left(\tau_{k+1}\right)$.

При обсуждении условий рекуррентности необходимо использование результатов, приведённых в $[28,29]$.

Для первого условия факторизации $\lambda_{1}\left[1-P_{0}\left(\lambda_{2} \mid \lambda_{1}\right)\right]=\lambda_{2}\left[1-P_{0}\left(\lambda_{1} \mid \lambda_{2}\right)\right]$ апостериорная вероятность $\mathrm{w}\left(\lambda_{1} \mid t\right)$ первого состояния процесса $\lambda(t)$ в момент $t_{k}$ наступления события потока имеет вид

$$
w\left(\lambda_{1} \mid t_{k}+0\right)=\frac{\left[\lambda_{1} P_{1}\left(\lambda_{1} \mid \lambda_{1}\right)-\lambda_{2} P_{1}\left(\lambda_{1} \mid \lambda_{2}\right)\right] w\left(\lambda_{1} \mid t_{k}-0\right)+\lambda_{2} P_{1}\left(\lambda_{1} \mid \lambda_{2}\right)}{\lambda_{2}\left[1-P_{0}\left(\lambda_{1} \mid \lambda_{2}\right)\right]} .
$$

Таким образом, апостериорная вероятность первого состояния процесса $\lambda(t)$ (несмотря на то, что поток рекуррентный и плотность $\left.p(\tau)=z_{1} e^{-z_{1} \tau}\right)$ зависит от предыстории, т.е. от значений апостериорной вероятности $w\left(\lambda_{1} \mid t\right)$ в моменты наступления событий $t_{1}, t_{2}, \ldots, t_{k}$. Если ввести дополнительное условие $\lambda_{1} P_{1}\left(\lambda_{1} \mid \lambda_{1}\right)=\lambda_{2} P_{1}\left(\lambda_{1} \mid \lambda_{2}\right)$, то

$$
w\left(\lambda_{1} \mid t_{k}+0\right)=\frac{P_{1}\left(\lambda_{1} \mid \lambda_{2}\right)}{1-P_{0}\left(\lambda_{1} \mid \lambda_{2}\right)}, \quad k=1,2, \ldots,
$$

т.е. апостериорная вероятность $w\left(\lambda_{1} \mid t\right)$ первого состояния процесса $\lambda(t)$ не будет зависеть от предыстории, а будет определяться лишь её значением в момент наступления события потока. Это значение апостериорной вероятности одинаково для всех моментов времени $t_{k}$ наступления событий потока, $k=1,2, \ldots$. Итак, при дополнительном ограничении имеется некоторая близость модулированного МАР-потока событий к простейшему потоку.

Для второго условия факторизации $\pi_{1}(0)\left(\lambda_{1}\left[1-P_{1}\left(\lambda_{1} \mid \lambda_{1}\right)\right]+\alpha_{1}\right)-\pi_{2}(0)\left(\lambda_{2}\left[1-P_{1}\left(\lambda_{2} \mid \lambda_{2}\right)\right]+\alpha_{2}\right)=0$ апостериорная вероятность $\mathrm{w}\left(\lambda_{1} \mid t\right)$ первого состояния процесса $\lambda(t)$ в момент $t_{k}$ также будет зависеть от предыстории, несмотря на то что поток рекуррентный и плотность экспоненциальная: $p(\tau)=z_{1} e^{-z_{1} \tau}, \tau \geq 0$.

Для третьего условия факторизации $P_{1}\left(\lambda_{1} \mid \lambda_{1}\right) P_{1}\left(\lambda_{2} \mid \lambda_{2}\right)-P_{1}\left(\lambda_{1} \mid \lambda_{2}\right) P_{1}\left(\lambda_{2} \mid \lambda_{1}\right)=0$ апостериорная вероятность $w\left(\lambda_{1} \mid t\right)$ первого состояния процесса $\lambda(t)$ в момент $t_{k}$ наступления события потока имеет вид

$$
w\left(\lambda_{1} \mid t_{k}+0\right)=\frac{P_{1}\left(\lambda_{1} \mid \lambda_{1}\right)}{P_{1}\left(\lambda_{1} \mid \lambda_{1}\right)+P_{1}\left(\lambda_{2} \mid \lambda_{1}\right)}=\pi_{1}(0), \quad k=1,2, \ldots .
$$

Таким образом, апостериорная вероятность $w\left(\lambda_{1} \mid t\right)$ не зависит от предыстории, а определяется лишь её (апостериорной вероятности) значением в момент наступления события потока. Итак, в данной ситуации имеется некоторая близость модулированного МАР-потока событий к простейшему потоку в том смысле, что апостериорная вероятность первого состояния процесса $\lambda(t)$ в моменты наступления событий потока принимает постоянное значение.

\section{Заключение}

Полученные результаты можно использовать для решения задачи оценивания неизвестных параметров модулированного МАР-потока событий, таких как интенсивности $\lambda_{1}, \lambda_{2}$ и вероятности $P_{0}\left(\lambda_{j} \mid \lambda_{i}\right)$, $P_{1}\left(\lambda_{j} \mid \lambda_{i}\right), i, j=1,2$. При этом, например, для нахождения оценок, после построения соответствующей функции правдоподобия, можно воспользоваться методом максимального правдоподобия либо применить метод моментов, решив соответствующие системы уравнений.

\section{ЛИТЕРАТУРА}

1. Kingman J.F.C. On doubly stochastic Poisson process // Proc. of Cambridge Phylosophical Society. 1964. V. 60, No. 4. P. $923-930$.

2. Башарин Г.П., Кокотушкин В.А., Наумов В.А. О методе эквивалентных замен расчета фрагментов связи // Известия АН СССР. Техническая кибернетика. 1980. № 6. С. 92-99.

3. Башарин Г.П., Кокотушкин В.А., Наумов В.А. О методе эквивалентных замен расчета фрагментов связи // Известия АН СССР. Техническая кибернетика. 1980. № 1. С. 55-61.

4. Neuts M.F. A versatile Markov point process // Journal of Applied Probability. 1979. V. 16. P. 764-779.

5. Дудин А.Н., Клименок В.И. Системы массового обслуживания с коррелированными потоками. Минск : Изд-во БГУ, 2000.175 с. 
6. Bushlanov I.V., Gortsev A.M., Nezhel'skaya L.A. Estimating parameters of the synchronous twofold-stochastic flow of events // Automation and Remote Control. 2008. V. 69, No. 9. P. 1517-1533.

7. Gortsev A.M., Nezhel'skaya L.A. Estimation of the dead time period and intensities of the synchronous double stochastic event flow // Radiotekhnika. 2004. No. 10. P. 8-16.

8. Василевская Т.П., Гориев А.М., Нежельская Л.А. Оценивание длительности мёртвого времени и параметров синхронного альтернирующего потока с проявлением либо непроявлением событий // Вестник Томского государственного университета. 2004. № 9 (II). С. 129-138.

9. Гориев А.М., Нежельская Л.А. Оценивание длительности мёртвого времени и параметров синхронного альтернирующего потока событий // Вестник Томского государственного университета. 2003. № 6. С. 232-239.

10. Гориев А.М., Нежельская Л.А. Оценивание параметров синхронного дважды стохастического потока событий методом моментов // Вестник Томского государственного университета. 2002. № 1(I). С. 24-29.

11. Gortsev A.M., Nezhel'skaya L.A. Estimation of the parameters of a synchro-alternating Poisson event flow by the method of moments // Radiotekhnika. 1995. V. 40, No. 7-8. P. 6-10.

12. Леонова М.А., Нежельская Л.А. Оценка максимального правдоподобия длительности мёртвого времени в обобщенном асинхронном потоке событий // Вестник Томского государственного университета. Управление, вычислительная техника и информатика. 2013. № 2 (23). С. 54-63.

13. Гориев А.М., Леонова М.А., Нежельская Л.А. Совместная плотность вероятностей длительности интервалов обобщенного асинхронного потока событий при непродлевающемся мёртвом времени // Вестник Томского государственного университета. Управление, вычислительная техника и информатика. 2012. № 4 (21). С. 14-25.

14. Леонова М.А., Нежельская Л.А. Вероятность ошибки при оценивании состояний обобщенного синхронного потока событий // Вестник Томского государственного университета. Управление, вычислительная техника и информатика. 2012. № 2 (19). C. 88-101.

15. Gortsev A.M., Nezhelskaya L.A. An asynchronous double stochastic flow with initiation of superfluous events // Discrete Mathematics and Applications. 2011. V. 21, No. 3. P. 283-290.

16. Гориев А.М., Нежельская Л.А. Оценивание параметров асинхронного потока с инициированием лишних событий методом моментов // Вестник Томского государственного университета. 2006. № 18. С. 267-273.

17. Gortsev A.M., Nezhel'skaya L.A., Shevchenko, T.I. Estimation of the states of an MC-stream of events in the presence of measurement errors // Russian Physics Journal. 1993. V. 36, No. 12. P. 1153-1167.

18. Калягин А.А., Нежельская Л.А. Оценка длительности мёртвого времени в обобщённом полусинхронном потоке событий // Новые информационные технологии в исследовании сложных структур : материалы Десятой рос. конф. с междунар. участием (9-13 июня 2014 г.). Томск : Издательский Дом Томского государственного университета, 2014. С. 96-97.

19. Гориев А.М., Калягин А.А., Нежельская Л.А. Оптимальная оценка состояний обобщённого полусинхронного потока событий // Вестник Томского государственного университета. Управление, вычислительная техника и информатика. 2010. № 2 (11). С. 66-81.

20. Гориев А.М., Нежельская Л.А. Полусинхронный дважды стохастический поток событий при продлевающемся мёртвом времени // Вычислительные технологии. 2008. Т. 13, № 1. С. 31-41.

21. Gortsev, A.M., Nezhel'skaya, L.A. Estimation of the dead-time period and parameters of a semi-synchronous double-stochastic stream of events // Measurement Techniques. 2003. V. 46, No. 6. P. 536-545.

22. Гориев А.М., Нежельская Л.А. Оценивание параметров полусинхронного дважды стохастического потока событий методом моментов // Вестник Томского государственного университета. 2002. № 1 (I). С. 18-23.

23. Нежельская Л.А. Оптимальное оценивание состояний полусинхронного потока событий в условиях его частичной наблюдаемости // Вестник Томского государственного университета. 2000. № 269. С.95-98.

24. Гориев А.М., Нежельская Л.А. О связи МС-потоков и МАР-потоков событий // Вестник Томского государственного университета. Управление, вычислительная техника и информатика. 2011. № 1 (14). С. 13-21.

25. Gortsev A.M., Nezhel'skaya L.A., Solov'ev A.A. Optimal state estimation in map event flows with unextendable dead time // Automation and Remote Control. 2012. V. 73, No. 8. P. 1316-1326.

26. Гориев А.М., Соловьев А.А. Совместная плотность вероятностей длительности интервалов МАР-потока событий и условия его рекуррентности // Вестник Томского государственного университета. Управление, вычислительная техника и информатика. 2012. № 3 (20). С. 32-41.

27. Нежельская Л.А. Апостериорные вероятности состояний модулированного МАР-потока событий // Новые информационные технологии в исследовании сложных структур : материалы Десятой рос. конф. с междунар. участием (9-13 июня 2014 г.). Томск : Издательский Дом Томского государственного университета, 2014. С. 95-96.

28. Нежельская Л.А. Оптимальная оценка состояний модулированного МАР-потока событий в условиях непродлевающегося мёртвого времени // Информационные технологии и математическое моделирование (ИТММ-2014) : материалы ХІІІ Междунар. науч.-практ. конф. им. А.Ф. Терпугова (20-22 ноября 2014 г.). Томск : Изд-во Том. ун-та, 2014. Ч. 2. С. $193-198$.

29. Nezhel'skaya L.A. Optimal State Estimation in Modulated MAP Event Flows with Unexendable Dead Time // Communications in Computer and Information Sciences : proceedings of the 13th International Scientific Conference ITMM 2014 named after A.F. Terpugov «Information Technologies and Mathematical Modeling» (November 20-22, 2014). Cham Heidelberg ; New York ; Dordrecht ; London : Springer, 2014. P. 342-350.

Нежельская Людмила Алексеевна, канд. техн. наук. E-mail: ludne@mail.ru Томский государственный университет 
Nezhelskaya Luydmila A. (Tomsk State University, Russian Federation).

Joint probability density of the intervals duration in modulated MAP event flows and its recurrence conditions.

Keywords: modulated MAP event flows; infinitesimal characteristics; probability density; joint probability density; flow recurrence conditions.

Consider a modulated MAP flow of events with the intensity represented by a piecewise constant random process $\lambda(t)$ with two states: $\lambda(t)=\lambda_{1}$ or $\lambda(t)=\lambda_{2}\left(\lambda_{1}>\lambda_{2} \geq 0\right)$. The time when the process $\lambda(t)$ remains at the $i$ th, $i=1,2$, state depends on two random values: 1) the first random value has the exponential distribution function $F_{i}^{(1)}(t)=1-e^{-\alpha_{i} t}, i=1,2$; when the $i$ th state ends, process $\lambda(t)$ transits with the probability equal one from the $i$ th state to the $j$ th, $i=1,2,(i \neq j) ; 2)$ the second random value has the exponential distribution function $F_{i}^{(2)}(t)=1-e^{-\lambda_{i} t}, i=1,2$; when the $i$ th state ends, process $\lambda(t)$ transits with probability $P_{1}\left(\lambda_{j} \mid \lambda_{i}\right)$ from the $i$ th state to the $j$ th $(i \neq j)$ and a flow event occurs or process $\lambda(t)$ transits with probability $P_{0}\left(\lambda_{j} \mid \lambda_{i}\right)$ from the $i$ th state to the $j$ th $(i \neq j)$, but the flow event does not occur, or process $\lambda(t)$ transits from the $i$ th state to the $i$ th with probability $P_{1}\left(\lambda_{i} \mid \lambda_{i}\right)$ and a flow event occurs. Here, $P_{1}\left(\lambda_{j} \mid \lambda_{i}\right)+P_{0}\left(\lambda_{j} \mid \lambda_{i}\right)+P_{1}\left(\lambda_{i} \mid \lambda_{i}\right)=1 ; i=1,2, i \neq j$.

The first and the second random values are independent from each other. Under these assumptions, $\lambda(t)$ is a Markov process. The infinitesimal characteristics matrices for the process $\lambda(t)$ are as follows:

$$
\begin{gathered}
\mathbf{D}_{0}=\left\|\begin{array}{cc}
-\left(\alpha_{1}+\lambda_{1}\right) & \alpha_{1}+\lambda_{1} P_{0}\left(\lambda_{2} \mid \lambda_{1}\right) \\
\alpha_{2}+\lambda_{2} P_{0}\left(\lambda_{1} \mid \lambda_{2}\right) & -\left(\alpha_{2}+\lambda_{2}\right)
\end{array}\right\|, \\
\mathbf{D}_{1}=\left\|\begin{array}{lc}
\lambda_{1} P_{1}\left(\lambda_{1} \mid \lambda_{1}\right) & \lambda_{1} P_{1}\left(\lambda_{2} \mid \lambda_{1}\right) \\
\lambda_{2} P_{1}\left(\lambda_{1} \mid \lambda_{2}\right) & \lambda_{2} P_{1}\left(\lambda_{2} \mid \lambda_{2}\right)
\end{array}\right\| .
\end{gathered}
$$

We consider the stationary operation mode for the flow. Denote by $\tau_{k}=t_{k+1}-t_{k}, k=1,2, \ldots$ the value of interval $k$ duration between the neighboring flow events. We may take that the probability density of the interval $k$ duration is $p\left(\tau_{k}\right)=p(\tau), \tau \geq 0$, for any $k$. Then, we can let $t_{k}=0$ without loss of generality, i.e., the moment of the event occurrence is $\tau=0$. Now, let $\left(t_{k}, t_{k+1}\right),\left(t_{k+1} t_{k+2}\right)$ be the neighboring intervals with the corresponding duration values $\tau_{k}=t_{k+1}-t_{k}, \tau_{k+1}=t_{k+2}-t_{k+1}$. Due to the stationary of the flow, the arrangement of the intervals on a time axis is arbitrarily. That is way, we may consider the neighboring intervals $\left(t_{1}, t_{2}\right),\left(t_{2}, t_{3}\right)$ with the corresponding duration values $\tau_{1}=t_{2}-t_{1}, \tau_{2}=t_{3}-t_{2} ; \tau_{1} \geq 0, \tau_{2} \geq 0$. Here $\tau_{1}=0$ corresponds to the moment $t_{1}$ and $\tau_{2}=0$ corresponds to the time moment $t_{2}$ when the next event in the flow occurs. The respective joint probability density is defined as $p\left(\tau_{1}, \tau_{2}\right), \tau_{1} \geq 0, \tau_{2} \geq 0$.

The aim of this article is to obtain the explicit form of the probability density $p(\tau)$ and the joint probability $p\left(\tau_{1}, \tau_{2}\right)$ and then to formulate the conditions of the flow recurrence. These formulas are obtained and are as follows:

$$
\begin{gathered}
p(\tau)=\gamma z_{1} e^{-z_{1} \tau}+(1-\gamma) z_{2} e^{-z_{2} \tau}, \quad \tau \geq 0, \\
\gamma=\frac{1}{z_{2}-z_{1}}\left\{z_{2}-\lambda_{1} \pi_{1}(0)\left[1-P_{0}\left(\lambda_{2} \mid \lambda_{1}\right)\right]-\lambda_{2} \pi_{2}(0)\left[1-P_{0}\left(\lambda_{1} \mid \lambda_{2}\right)\right]\right\}, \\
z_{1}=\frac{1}{2}\left[\left(\lambda_{1}+\lambda_{2}+\alpha_{1}+\alpha_{2}\right)-\sqrt{\left(\lambda_{1}-\lambda_{2}+\alpha_{1}-\alpha_{2}\right)^{2}+4\left(\alpha_{1}+\lambda_{1} P_{0}\left(\lambda_{2} \mid \lambda_{1}\right)\right)\left(\alpha_{2}+\lambda_{2} P_{0}\left(\lambda_{1} \mid \lambda_{2}\right)\right)}\right], \\
z_{2}=\frac{1}{2}\left[\left(\lambda_{1}+\lambda_{2}+\alpha_{1}+\alpha_{2}\right)+\sqrt{\left(\lambda_{1}-\lambda_{2}+\alpha_{1}-\alpha_{2}\right)^{2}+4\left(\alpha_{1}+\lambda_{1} P_{0}\left(\lambda_{2} \mid \lambda_{1}\right)\right)\left(\alpha_{2}+\lambda_{2} P_{0}\left(\lambda_{1} \mid \lambda_{2}\right)\right)}\right], 0<z_{1}<z_{2}, \\
\pi_{1}(0)=\left\{\lambda_{2} P_{1}\left(\lambda_{1} \mid \lambda_{2}\right)\left(\lambda_{1}+\alpha_{1}\right)+\lambda_{1} P_{1}\left(\lambda_{1} \mid \lambda_{1}\right)\left(\alpha_{2}+\lambda_{2} P_{0}\left(\lambda_{1} \mid \lambda_{2}\right)\right)\right\}\left\{\lambda_{1} P_{1}\left(\lambda_{2} \mid \lambda_{1}\right)\left(\lambda_{2}+\alpha_{2}\right)+\right. \\
\left.+\lambda_{2} P_{1}\left(\lambda_{1} \mid \lambda_{2}\right)\left(\lambda_{1}+\alpha_{1}\right)+\lambda_{1} P_{1}\left(\lambda_{1} \mid \lambda_{1}\right)\left(\alpha_{2}+\lambda_{2} P_{0}\left(\lambda_{1} \mid \lambda_{2}\right)\right)+\lambda_{2} P_{1}\left(\lambda_{2} \mid \lambda_{2}\right)\left(\alpha_{1}+\lambda_{1} P_{0}\left(\lambda_{2} \mid \lambda_{1}\right)\right)\right\}, \\
\pi_{2}(0)=\left\{\lambda_{1} P_{1}\left(\lambda_{2} \mid \lambda_{1}\right)\left(\lambda_{2}+\alpha_{2}\right)+\lambda_{2} P_{1}\left(\lambda_{2} \mid \lambda_{2}\right)\left(\alpha_{1}+\lambda_{1} P_{0}\left(\lambda_{2} \mid \lambda_{1}\right)\right)\right\}\left\{\lambda_{1} P_{1}\left(\lambda_{2} \mid \lambda_{1}\right)\left(\lambda_{2}+\alpha_{2}\right)+\right. \\
\left.+\lambda_{2} P_{1}\left(\lambda_{1} \mid \lambda_{2}\right)\left(\lambda_{1}+\alpha_{1}\right)+\lambda_{1} P_{1}\left(\lambda_{1} \mid \lambda_{1}\right)\left(\alpha_{2}+\lambda_{2} P_{0}\left(\lambda_{1} \mid \lambda_{2}\right)\right)+\lambda_{2} P_{1}\left(\lambda_{2} \mid \lambda_{2}\right)\left(\alpha_{1}+\lambda_{1} P_{0}\left(\lambda_{2} \mid \lambda_{1}\right)\right)\right\} \\
p\left(\tau_{1}, \tau_{2}\right)=p\left(\tau_{1}\right) p\left(\tau_{2}\right)+\gamma(1-\gamma) \frac{\lambda_{1} \lambda_{2}}{z_{1} z_{2}}\left[P_{1}\left(\lambda_{1} \mid \lambda_{1}\right) P_{1}\left(\lambda_{2} \mid \lambda_{2}\right)-P_{1}\left(\lambda_{1} \mid \lambda_{2}\right) P_{1}\left(\lambda_{2} \mid \lambda_{1}\right)\right] \times \\
\times\left(z_{1} e^{-z_{1} \tau_{1}}-z_{2} e^{\left.-z_{2} \tau_{1}\right)}\right)\left(z_{1} e^{-z_{1} \tau_{2}}-z_{2} e^{\left.-z_{2} \tau_{2}\right)}, \quad \tau_{1} \geq 0, \tau_{2} \geq 0 .\right.
\end{gathered}
$$

\section{REFERENCES}

1. Kingman J.F.C. On doubly stochastic Poisson process. Proc. of Cambridge Phylosophical Society, 1964, vol. 60, no. 4, pp. 923-930.

2. Basharin G.P., Kokotushkin V.A., Naumov V.A. O metode ekvivalentnykh zamen rascheta fragmentov svyazi [On a method of equivalent substitutions for communications network fragments calculation]. Izvestiya AN SSSR. Tekhnicheskaya kibernetika, 1980, no. 6 , pp. 92-99. 
3. Basharin G.P., Kokotushkin V.A., Naumov V.A. O metode ekvivalentnykh zamen rascheta fragmentov svyazi [On a method of equivalent substitutions for communications network fragments calculation]. Izvestiya AN SSSR. Tekhnicheskaya kibernetika, 1980, no. 1 , pp. 55-61.

4. Neuts M.F. A versatile Markovian point process. Journal of Applied Probability, 1979, vol. 16, pp. 764-779. DOI: 10.2307/3213143

5. Dudin A.N., Klimenok V.I. Sistemy massovogo obsluzhivaniya s korrelirovannymi potokami [Queueing systems with correlated flows]. Minsk: BGU Publ., 2000. 175 p.

6. Bushlanov, I.V., Gortsev, A.M., Nezhel'skaya, L.A. Estimating parameters of the synchronous twofold-stochastic flow of events. Automation and Remote Control, 2008, vol. 69, no. 9, pp. 1517-1533. DOI: 10.1134/S0005117908090075

7. Gortsev, A.M., Nezhel'skaya, L.A. Estimation of the dead time period and intensities of the synchronous double stochastic event flow. Radiotekhnika, 2004, no. 10, pp. 8-16.

8. Vasilevskaya T.P., Gortsev A.M., Nezhel'skaya L.A. Estimation of the dead-time period and parameters of the synchronous alternative flow with displaying or no displaying of events. Vestnik Tomskogo gosudarstvennogo universiteta - Tomsk State University Journal, 2004, no. 9 (II), pp. 129-138. (In Russian).

9. Gortsev A.M., Nezhel'skaya L.A. Otsenivanie dlitel'nosti mertvogo vre $\neg$ meni i parametrov sinkhronnogo al'terni $\neg$ ruyushchego potoka sobytiy [Estimation of the dead-time period and parameters of the synchronous alternative flow of events]. Vestnik Tomskogo gosudarstvennogo universiteta - Tomsk State University Journal, 2003, no. 6, pp. 232-239.

10. Gortsev A.M., Nezhel'skaya L.A. Otsenivanie parametrov sinkhronnogo dvazhdy stokhasticheskogo potoka sobytiy metodom momentov [Estimating parameters of the synchronous twofold-stochastic flow of events by the moment method]. Vestnik Tomskogo gosudarstvennogo universiteta - Tomsk State University Journal, 2002, no. 1(I), pp. 24-29.

11. Gortsev, A.M., Nezhel'skaya, L.A. Estimation of the parameters of a synchro-alternating Poisson event flow by the method of moments. Radiotekhnika, 1995, vol. 40, no. 7-8, pp. 6-10.

12. Leonova M.A., Nezhel'skaya L.A. Maximum-likelihood estimation of the dead time in a generalized asynchronous flow of events. Vestnik Tomskogo gosudarstvennogo universiteta - Tomsk State University Journal, 2013, no. 2 (23), pp. 54-63. (In Russian).

13. Gortsev A.M., Leonova M.A., Nezhel'skaya L.A. Joint probability density of the intervals duration for generalized asynchronous flow of events with unextendable dead time. Vestnik Tomskogo gosudarstvennogo universiteta - Tomsk State University Journal, 2012, no. 4 (21), pp. 14-25. (In Russian).

14. Leonova M.A., Nezhel'skaya L.A. The probability of wrong decision in the estimation of states of a generalized asynchronous flow of events. Vestnik Tomskogo gosudarstvennogo universiteta - Tomsk State University Journal, 2012, no. 2 (19), pp. 88-101. (In Russian).

15. Gortsev, A.M., Nezhelskaya, L.A. An asynchronous double stochastic flow with initiation of superfluous events. Discrete Mathematics and Applications, 2011, vol. 21, no. 3, pp. 283-290. DOI: 10.4213/dm1 141

16. Gortsev A.M., Nezhel'skaya L.A. Otsenivanie parametrov asinkhronnogo potoka s initsiirovaniem lishnikh sobytiy metodom momentov [Estimating parameters of the asynchronous flow with initiation of superfluous events by moment method]. Vestnik Tomskogo gosudarstvennogo universiteta - Tomsk State University Journal, 2006, no. 18, pp. 267-273.

17. Gortsev, A.M., Nezhel'skaya, L.A., Shevchenko, T.I. Estimation of the states of an MC-stream of events in the presence of measurement errors. Russian Physics Journal, 1993, vol. 36, no. 12, pp. 1153-1167.

18. Kalyagin A.A., Nezhel'skaya L.A. [Estimation of the dead time duration in a generalized semi-synchronous flow of events]. Materialy konferentsii "Novye informatsionnye teknologii v issledovanii slozhnykh struktur" [Proceedings of the 10th Russian conference with international participation "Novel information technologies for studying complex structures"]. Tomsk: Tomsk State University Publ., 2014, pp. 96-97. (In Russian).

19. Gortsev A.M., Kalyagin A.A., Nezhel'skaya L.A. Optimal state estimation for a generalized semi-synchronous flow of events. Vestnik Tomskogo gosudarstvennogo universiteta. Upravlenie, vychislitelnaya tekhnika I informatika - Tomsk State University Journal of Control and Computer Science, 2010, no. 2 (11), pp. 66-81. (In Russian).

20. Gortsev A.M., Nezhel'skaya L.A. Semisynchronous double stochastic flow of events when the dead time is prolonged. Vychislitel'nye tekhnologii - Computational Technologies, 2008, vol. 13, no. 1, pp. 31-41. (In Russian).

21. Gortsev A.M., Nezhel'skaya L.A. Estimation of the dead-time period and parameters of a semi-synchronous double-stochastic stream of events. Measurement Techniques, 2003, vol. 46, no. 6, pp. 536-545. DOI: 10.1023/A:1025499509015

22. Gortsev A.M., Nezhel'skaya L.A. Otsenivanie parametrov polusinkhronnogo dvazhdy stokhasticheskogo potoka sobytiy metodom momentov [Estimating parameters of the semi-synchronous double stochastic flow of events by moment method]. Vestnik Tomskogo gosudarstvennogo universiteta. Upravlenie, vychislitelnaya tekhnika I informatika - Tomsk State University Journal of Control and Computer Science, 2002, no. 1 (I), pp. 18-23.

23. Nezhel'skaya L.A. Optimal'noe otsenivanie sostoyaniy polusinkhronnogo potoka sobytiy v usloviyakh ego chastichnoy nablyudaemosti [Optimal state estimation of the semi-synchronous flow of events under incomplete observability]. Vestnik Tomskogo gosudarstvennogo universiteta - Tomsk State University Journal, 2000, no. 269, pp.95-98.

24. Gortsev A.M., Nezhel'skaya L.A. On connection of MC flows and MAP flows of events. Vestnik Tomskogo gosudarstvennogo universiteta. Upravlenie, vychislitelnaya tekhnika I informatika - Tomsk State University Journal of Control and Computer Science, 2011, no. 1 (14), pp. 13-21. (In Russian).

25. Gortsev A.M., Nezhel'skaya L.A., Solov'ev A.A. Optimal state estimation in map event flows with unextendable dead time. Automation and Remote Control, 2012, vol. 73, no. 8, pp. 1316-1326. DOI: 10.1134/S000511791208005X

26. Gortsev A.M., Solov'ev A.A. The join density of probability intervals MAP of the flow of events and condition of its recurrence. Vestnik Tomskogo gosudarstvennogo universiteta. Upravlenie, vychislitelnaya tekhnika I informatika - Tomsk State University Journal of Control and Computer Science, 2012, no. 3 (20), pp. 32-41. (In Russian). 
27. Nezhel'skaya L.A. [Optimal state estimation for a modulated MAP events flow with unextendable dead time]. Materialy XIII mezhdunarodnoi nauchno-prakticheskoi konferentsii "Informatsionnye teknologii i matematicheskoe modelirovanie (ITMM)" [Proceedings of the $13^{\text {th }}$ International scientific conference named after A.F. Terpugov "Information Technologies and Mathematical Modeling"]. Tomsk: Tomsk State University Publ., 2014, pt. 2, pp. 193-198. (In Russian).

28. Nezhel'skaya L.A. [Optimal State Estimation in Modulated MAP Event Flows with Unexendable Dead Time]. Informatsionnye tekhnologii i matematicheskoe modelirovanie (ITMM-2014): materialy XIII Mezhdunarodnoy nauchno-prakticheskoy konferentsii [Proceedings of the 13th International scientific conference named after A.F. Terpugov "Information Technologies and Mathematical Modeling"]. Tomsk: Tomsk State University Publ., 2014, pt. 2, pp. 193-198. (In Russian).

29. Nezhel'skaya, L.A. [Optimal State Estimation in Modulated MAP Event Flows with Unexendable Dead Time]. Communications in Computer and Information Sciences: Proceedings of the 13th International Scientific Conference ITMM 2014 named after A.F. Terpugov “Information Technologies and Mathematical Modeling”. Cham Heidelberg New York Dordrecht London: Springer, 2014, pp. 342-350. 